\title{
Clinical Significance of Combined Anti-Cyclic Citrullinated Peptide Antibody and Rheumatoid Factor Assays in Rheumatoid Arthritis Diagnosis
}

\author{
Qingmei CAO, Yanfeng WANG, Yuanyuan JING \\ Department of Laboratory Medicine, Yanan University Affiliated Hospital, Yanan, China
}

\begin{abstract}
Objectives: This study aims to investigate the value of combined anti-cyclic citrullinated peptide (CCP) antibody and rheumatoid factor (RF) assays in the clinical diagnosis of patients with rheumatoid arthritis (RA).

Patients and methods: Results of both anti-CCP and RF detection in 70 RA patients ( 13 males, 57 females; mean age $49.2 \pm 12.1$ years; range 35 to 61 years) and 112 non-RA patients ( 35 males, 77 females; mean age $42.5 \pm 7.1$ years; range 35 to 49 years) were retrospectively and statistically analyzed. Results: In RA patients, positive rates of anti-CCP and RF were $70.0 \%$ and $77.1 \%$, respectively. These rates were significantly higher than the rates in non-RA patients $(2.7 \%$ and $4.5 \%$, respectively; $\mathrm{p}<0.05)$. The sensitivity of combined anti-CCP and RF assays was $87.1 \%$ compared to the sensitivities of a single item assay of $70 \%$ and $77.1 \%$ for anti-CCP and RF, respectively. However, the combined assay had slightly lower specificity. With respect to the diagnostic index and rate of total consistency, the combined assay was superior to any single item assay.

Conclusion: Combined anti-CCP and RF assays are more valuable in diagnosing RA than any single item assay.

Keywords: Cyclic citrullinated peptide; rheumatoid arthritis; rheumatoid factor.
\end{abstract}

Rheumatoid arthritis (RA) is a common autoimmune disease, affecting approximately 1\% of all people worldwide and the pathological characteristics of RA can lead to disability. ${ }^{1}$ There are no typical early clinical manifestations to facilitate diagnosis, which often leads to misdiagnosis or no diagnosis at all. The prognosis for RA patients is poor, and may include joint dysfunction and loss of ability to work, and may even include life-threatening vasculitis of the main organs. ${ }^{2}$ Therefore, early diagnosis and treatment are essential. Recent studies have begun to explore the use of combined assays in RA diagnosis in China. In a study, it was reported that combined assays of anti-cyclic citrullinated peptide (CCP) antibody and anti-keratin antibody, anti-perinuclear factor and anti-CCP antibody, and anti-perinuclear factor and anti-keratin antibody show high specificities of $98.9 \%, 98.9 \%$, and $100 \%$, respectively. ${ }^{3}$ However, single item assays are unable to evaluate both sensitivity and specificity. Specificity, also known as the negative rate, indicates the diagnostic accuracy in healthy people who were not diagnosed with the disease according to diagnostic standards, which reflects the ability of a test to verify non-patients. Sensitivity indicates the probability of a false negative during diagnosis, while specificity indicates the probability of a false positive. Therefore, most investigators conduct combinations of various clinical assays to improve early RA diagnosis. However, the evaluation of diagnostic RA detection methods is incomplete and requires further study. Cyclin-dependent kinase 2, rheumatoid factor (RF), immunoglobulin $M$ (IgM)-RFs, IgA-RFs, IgG-RFs, and anti-CCP all have a certain diagnostic significance when comparing various RA subgroups. $\mathrm{RF}$ is an

Received: November 16, 2013 Accepted: August 01, 2014 Published online: April 06, 2015

Correspondence: Qingmei Cao, M.D. Department of Laboratory Medicine, Yanan University Affiliated Hospital, 716000 Yanan, China.

Tel: +86-0911-211 1898 e-mail: qingmeicao@163.com

(2015 Turkish League Against Rheumatism. All rights reserved. 
autoantibody that recognizes denaturized IgG, which is found in the serum of RA patients. RF can bind to the fragment crystallizable portion of $\mathrm{IgG}$, and is mainly detected in serum and joint fluid as an antibody of contravariance. $\mathrm{CCP}$, which is a peptide fragment of annular silk protein, is recognized by anti-CCP, a type of IgG antibody that has high sensitivity and specificity. RA patients with anti-CCP have more severe bone damage than those negative for anti-CCP antibody. In 1998, researchers described antiCCP for the first time, showed that tests for anti-CCP are highly sensitive and specific for $\mathrm{RA}$, and considered that it may be a novel serum marker for the disease. However, although various qualitative indicators show high specificity, the sensitivities of these indicators are not ideal. Each single indicator has advantages and shortcomings in RA diagnosis, so no single indicator appears to be ideal. Therefore, in this study, we aimed to investigate the value of combined anti- CCP antibody and RF assays in the clinical diagnosis of patients with RA.

\section{PATIENTS AND METHODS}

Rhuematoid arthritis was diagnosed according to the RA classification diagnostic standards revised by the American Rheumatism Association in 1987, which is believed to be the "gold standard." Specifically, there are seven terms in the standard: (i) Morning stiffness for at least $1 \mathrm{~h}$ ( $\geq 6$ weeks), (ii) three or more damaged joints ( $\geq 6$ weeks), (iii) damaged joints of the hands (wrist, metacarpophalangeal, or proximal interphalangeal joints; $\geq 6$ weeks), (iv) symmetric arthritis ( $\geq 6$ weeks), (v) subcutaneous rheumatoid nodules, (vi) radiographic changes, and (vii) serum positive for RF (titer >1:32). Patients were diagnosed with RA if four of the seven terms were detected. Based on these criteria, patients who were not diagnosed with RA and who were negative for anti-CCP were included as the controls. Most patients in the control group were diagnosed with osteoarthritis or ankylosing spondylitis, while a small number were diagnosed with connective tissue diseases.

A total of 182 patients who visited the Department of Rheumatism, Affiliated Hospital of Yan'an University between May 2011 and October
2012 were selected and classified according to the criteria. Among them, 70 patients (13 males, 57 females; mean age 49.2 \pm 12.1 years; range 35 to 61) were diagnosed with RA. Male to female ratio was 13:57. The non-RA group included 112 patients (35 males, 77 females; mean age $42.5 \pm 7.1$ years; range 35 to 49 years). Male to female ratio was $5: 11$. The reagents for the antiCCP assay were from EUROIMMUN AG (Lübeck, Schleswig-Holstein, Germany). A Multiskan MK3 model Microplate Reader (Shanghai Shiye Kehua Biochemicals Co., Ltd., China) was used. Anti-CCP was detected using an enzyme-linked immunosorbent assay. RF assay reagents were from Shenzhen Goldsite Biotechnology Co., Ltd. (Shenzhen, Guangdong, China), and a specific protein analyzer was used (Tianjin Meide Pacific Technology Ltd., China). RF was detected using a rate nephelometric assay.

Venous blood ( $3 \mathrm{~mL}$ ) was drawn from the fasting subjects in the morning and placed into heparinized anti-coagulant tubes, and then centrifuged at $2136 \times \mathrm{g}$ to obtain blood plasma for the anti-CCP and RF assays. All tests were conducted in strict accordance to the standard operating procedures.

\section{Statistical analysis}

Statistical analysis was performed using the SPSS version 13.0 software program (SPSS Inc., Chicago, IL, USA). Enumeration data were described as positive rate, and the McNemar's test was used to estimate the difference between the rates, with $p<0.05$ considered statistically significant. Sensitivity, specificity, total rate consistency $(\pi)$, and diagnosis index were calculated. The sensitivity of the combined assay was estimated using sensitivities of $A$ and $B$ : (sensitivity of $A$ ) + (sensitivity of $B) *[1$ - (sensitivity of $A)]$.

\section{RESULTS}

The positive rate of anti-CCP in the $70 \mathrm{RA}$ patients was $70.0 \%$ (49/70), significantly higher than that in the non-RA patients $(2.7 \% ; 3 / 112)$ (Table 1). This difference was statistically significant $\left(\chi^{2}=12.041, \mathrm{p}<0.05\right)$.

The positive rate of $\mathrm{RF}$ in the $70 \mathrm{RA}$ patients was $77.1 \%$ (54/70), significantly higher than that in 
Table 1. Results of anti-cyclic citrullinated peptide antibody serum assay in study patients

\begin{tabular}{|c|c|c|c|c|c|}
\hline \multirow[t]{2}{*}{ Anti-CCP } & \multicolumn{2}{|c|}{ Gold standard } & \multirow[b]{2}{*}{ Total } & \multirow[b]{2}{*}{$\chi^{2}$} & \multirow[b]{2}{*}{$p$} \\
\hline & $\mathrm{RA}$ & Non-RA & & & \\
\hline+ & 49 & 3 & 52 & \multirow{2}{*}{12.041} & \multirow{2}{*}{$<0.05$} \\
\hline- & 21 & 109 & 130 & & \\
\hline Total & 70 & 112 & 182 & - & - \\
\hline
\end{tabular}

the non-RA patients (4.5\%; 5/112) (Table 2). The difference was statistically significant $\chi^{2}=4.762$, $\mathrm{p}<0.05)$.

Sensitivity was significantly increased and specificity was lower in the combined assays (Table 3). Additionally, the total rate consistency and diagnosis index were superior in combined assays than any single item assay.

\section{DISCUSSION}

Rhuematoid arthritis is a symmetrical inflammatory form of arthritis mainly involving small joints of the hands and feet. Patients presenting with a systemic immune response not only have RA, but also rheumatoid disease. RA lesions are not limited to the joints, and the lungs are commonly involved. Lung involvement rates in RA differ in various reports and the incidence rates reported in the literature range from $1.6 \%$ to $40 \%{ }^{4} \mathrm{RA}$ can occur at any age, and the peak incidence is in people aged 35-45 years and those aged over 60 years. The worldwide annual incidence rate is approximately 1-3\%. Previously, RF detection and radiography were considered conventional indicators for the auxiliary diagnosis of RA. Although RF sensitivity is high for RA, the specificity is low and radiographic changes were most apparent after joint damage. ${ }^{5}$ Therefore, indicators such as anti-CCP and other methods
Table 2. Results of rheumatoid factor detected by rate nephelometric assay in rheumatoid arthritis diagnosis

\begin{tabular}{lcccccc}
\hline RF & \multicolumn{3}{c}{ Gold standard } & & \\
\cline { 2 - 6 } & RA & Non-RA & Total & $\chi^{2}$ & $p$ \\
\hline+ & 54 & 5 & 59 & 4.762 & $<0.05$ \\
- & 16 & 107 & 123 & & \\
Total & 70 & 112 & 182 & - & - \\
\hline RF: Rheumatoid factor; RA: Rheumatoid arthritis.
\end{tabular}

have gradually been introduced in the clinic for the auxiliary diagnosis of RA.

Anti-CCP is currently accepted as an important indicator for the early diagnosis and screening of RA, with a sensitivity and specificity of $68 \%$ and $98 \%$, respectively, which is consistent with the findings in this study. It was reported ${ }^{6}$ that tests for the anti-CCP antibody had better sensitivity and specificity in diagnosing RA. Anti-CCP can appear in the early stages of RA and have an important role in early RA diagnosis. ${ }^{7,8}$ Some studies suggested that the anti-CCP antibody assay plays an important role in diagnosing $\mathrm{RA}$ patients who are RF-negative and have atypical clinical symptoms. ${ }^{9,10} \mathrm{RF}$ is the only serological criterion in the standard revised RA diagnosis from the American Rheumatism Association and it has a higher positive rate in patients with other autoimmune diseases, such as systemic lupus erythematosus, sicca syndrome, autoimmune hepatitis, and osteoarthritis, and even in patients with infectious diseases or healthy individuals. Therefore, RF assays lack diagnostic specificity, which is not conducive to making an early RA diagnosis. The anti-CCP antibody is specifically secreted by $B$ lymphocytes in patients with $\mathrm{RA}$, while $\mathrm{B}$ lymphocytes in patients with other diseases or in healthy people do not secrete anti$\mathrm{CCP}$. Testing positive for anti-CCP is associated with RA progression, since secretion of the $\mathrm{CCP}$ antigen promotes the immune system to release anti-CCP antibodies. Therefore, tests for the

Table 3. Actual results of single assay and combination assay of various indicators in rheumatoid arthritis diagnosis

\begin{tabular}{|c|c|c|c|c|}
\hline \multirow[t]{2}{*}{ Detection indicator } & Sensitivity & Specificity & Total consistent rate & Diagnosis index \\
\hline & $\%$ & $\%$ & $\%$ & $\%$ \\
\hline Anti-cyclic citrullinated peptide & 70.0 & 97.3 & 86.8 & 67.3 \\
\hline Rheumatoid factor & 77.1 & 95.5 & 88.5 & 72.6 \\
\hline Anti-cyclic citrullinated peptide + rheumatoid factor & 93.1 & 94.6 & 91.8 & 81.7 \\
\hline
\end{tabular}


anti-CCP antibody have a high specificity, ${ }^{11,12}$ and anti-CCP is an autoantibody highly specific to $\mathrm{RA}$, making it a new diagnostic serum biomarker for RA. ${ }^{13}$ While the high specificity of anti-CCP might appear to provide a definitive RA diagnosis, several non-RA cases were anti-CCP positive. This could be explained by several possible situations: (i) non-specific reverse operations easily generated in bodies of some persons with special constitution, allergic constitution and senile osteoporosis, (ii) cross-contamination during the assays, specimen hemolysis, bacterial contamination, or other technical problems, or (iii) issues with the reagents, as it is difficult to achieve perfect accuracy with any kind of reagent. However, most patients negative for anti-CCP are likely to suffer from osteoarthritis or ankylosing spondylitis, while others suffer from systemic lupus erythematosus, sicca syndrome, or other related diseases. Our results are consistent with results from tests for antiCCP showing high sensitivity, low specificity, and susceptibility to interference from other inflammatory factors in RA. ${ }^{14}$

Since early diagnosis and treatment are crucial for a positive prognosis in RA, the sensitivity of the indicator used for detection is very important. In some domestic reports, the parallel combination mode was typically used to calculate the sensitivity of the combined assay. Thus, the combination of highly specific anti-CCP and highly sensitive RF assays is widely used. At present, the anti-CCP antibody assay has gradually become widely used in the clinic. In relevant domestic reports, ${ }^{14,15}$ the combination of anti-CCP antibody and $\mathrm{RF}$ assays was superior to either single item assay. There was no difference due to sex, which is consistent with the report that antiCCP antibodies can act as an early diagnostic indicator of RA. Therefore, the combined assay had a higher diagnostic value when combined with the RF assay. According to Table 3, the sensitivity of the combined assay is $87.1 \%$, higher than that of anti-CCP or RF assays alone (70.0\% or $77.1 \%$, respectively), whereas the combined assay specificity is $94.6 \%$, slightly lower than that of anti-CCP (97.3\%) or RF $(95.5 \%)$ alone. In addition, the total consistency rate (91.8\%) and diagnosis index (81.7\%) are higher for the combined assay than for either single item assay. Therefore, we suggest that the combined anti-CCP and RF assay is necessary in the clinic, and has applicable value in early diagnosis, early treatment, and prevention of $\mathrm{RA}$. In summary, the combination of anti-CCP and RF assays greatly increases RA diagnosis sensitivity and makes up for the shortcomings of single item assays, providing an experimental method for early diagnosis and treatment in the clinic. Nevertheless, further studies are required to investigate the value of different combinations of the anti-CCP antibody assay and assays for various subtypes of RF in RA diagnosis.

\section{Declaration of conflicting interests}

The authors declared no conflicts of interest with respect to the authorship and/or publication of this article.

\section{Funding}

The authors received no financial support for the research and/or authorship of this article.

\section{REFERENCES}

1. Su Q, Wang Y, Wang YN, Wei X. Clinical significance of combined detection of Anti- CCP antibody and RF in rheumatoid arthritis. China Healthcare Innovation 2011;6:69.

2. Luo MF, Wang F. Three Bi Tang combined with methotrexate, sulfasalazine in treatment of rheumatoid arthritis. J Chin Practical Diagnosis and Therapy 2010;24:516-7.

3. Sun YL, Ma Y, Li XL. The application value of RF, Anti -CCP antibody, AFP, AKA levels in the diagnosis of RA. J Radioimmunol 2011;24:450-1.

4. Winkler G, Kempler P. Pathomechanism of diabetic neuropathy: background of the pathogenesis-oriented therapy. Orv Hetil 2010;151:971-81.

5. Xia WJ, Liu YQ, Cong L, Luo PT. Anti-CCP and RF in the diagnosis of rheumatoid arthritis. China Foreign Medical Treatment 2011;20:9-10.

6. Xia WJ, Liu YQ, Cong L, Luo PT. Analysis of the clinical application of Anti-CCP diagnosis of rheumatoid arthritis. Int J Laboratory Med 2011;32:120-1.

7. Dai WL, Chen ZZ. The diagnostic role of combined detection of Anti-CCP antibody and RF in RA. Exp Laboratory Med 2009;27:379-80.

8. Meng Y, Wang YY, Zhao CM. Anti-CCP study of the relationship between antibody and rheumatoid arthritis. J Xinjiang Med Univ 2010;3:558-60.

9. Li JS. ELISA method was used to detect the significance of Anti- CCP antibody in rheumatoid arthritis. Modern Preventive Medicine 2010;37:3329. 
10. Yu YX, Chi SH, Yang S. Study on the relationship between serum Anti -cyclic citrullinated peptide antibody and rheumatoid arthritis. China J Modern Med 2011;21:148.

11. Ye JR, Yuan LQ. Comparison of Anti-CCP antibody and RF in patients with rheumatoid arthritis diagnosis. J Med Res 2010;39:105-8.

12. Du J, Guo JL, Li ZS. Clinical significance of detection of Anti -CCP antibody in early rheumatoid arthritis. Chin J Laboratory Diagnosis 2009;13:506-9.
13. Ren CF, Wang QX, Li XW. The relationship between Anti-CCP detection and other laboratory indexes in patients with rheumatoid arthritis. Shandong Med J 2010;50:71-3.

14. Zhang $\mathrm{J}$, Li Z. Retrospective analysis of relevant indicators of rheumatoid arthritis. Chin J Modern Drug Application 2010;4:19-20.

15. Wang HZ, Ma FF, Xie ZJ. Clinical diagnostic value of four indexes of rheumatoid arthritis. Clin $\mathrm{J}$ Medical Officer 2011:39:782-4. 